\title{
Comparação das Respostas Fisiológicas, Perceptuais e Afetivas Durante Caminhada em Ritmo Autosselecionado por Mulheres Adultas de Três Diferentes Faixas Etárias
}

\author{
Comparison of the Physiological, Perception and Affective \\ Responses During Treadmill Walking at Self-Selected Pace by \\ Adult Women of Three Different Age Groups
}

Cosme Franklim Buzzachera ${ }^{1,2}$
Carlo Baldari
Hassan Mohamed Elsangedy ${ }^{2}$
Kleverton Krinski $^{1}$
Bruno Vinicius Santos ${ }^{1}$
Wagner De Campos ${ }^{1}$
Laura Guidetti $^{2}$
Sergio Gregorio Da Silva ${ }^{1}$

1. Departamento de Educação Física, Universidade Federal do Paraná, Curitiba, Paraná, Brasil.

2. Dipartimento di Scienze della Salute, Università degli Studi di Roma "Foro Italico", Roma, Itália.

Endereço para correspondência: Sergio Gregorio da Silva Rua Coração de Maria, 92, Jardim Botânico

CEP: 80.215-370

Curitiba - Paraná

E-mail: sergiogregorio@ufpr.br

\begin{abstract}
OBJETIVO
Comparar as respostas fisiológicas, perceptuais e afetivas durante caminhada em ritmo autosselecionado por mulheres adultas de três diferentes faixas etárias. Métodos: Foram investigados 66 sujeitos do sexo feminino, previamente sedentários, distribuídos de acordo com a sua idade cronológica nos seguintes grupos: GI (20,0-25,0 anos, $n=22)$, GII (30,0-35,0 anos, $n=22)$ e GIII (40,0-45,0 anos, $n=22)$. Todos os participantes foram submetidos a (i) pré-avaliação médica, avaliação antropométrica e processo de familiarização, (ii) teste incremental máximo em esteira, e (iii) um teste de 20-minutos de caminhada em ritmo autosselecionado em esteira. As respostas fisiológicas (consumo de oxigênio, $\mathrm{O} 2$, e frequência cardíaca, FC) foram mensuradas continuamente durante a realização do teste de 20-minutos de caminhada em ritmo autosselecionado. Por sua vez, as respostas perceptuais (percepção subjetiva de esforço, PSE) e afetivas foram determinadas a cada intervalo de cinco minutos do teste. Para a análise estatística, empregou-se uma ANOVA de um fator (faixa etária), adotando-se um valor de $p<0,05$. Resultados: Nenhuma diferença significativa foi verificada entre os grupos etários no $\mathrm{O} 2$ e na FC durante teste de 20-minutos de caminhada em ritmo autosselecionado. A velocidade de caminhada também foi similar entre os grupos etários. Contudo, as respostas fisiológicas relativas aos valores máximos (\%O2 $2_{\text {Máx }}$ e \%FC $C_{\text {Máx }}$ ) e aos valores no limiar ventilatório ( $(\mathrm{LV}$ \% O2LV e \%FCLV) foram mais elevadas em GIII comparativamente à Gl e GII $(p<0,05)$. Nenhuma diferença foi verificada tanto na PSE como no afeto entre os grupos etários. Conclusão: As respostas fisiológicas, porém não as respostas perceptuais e afetivas, diferem de acordo com a faixa etária investigada durante realização de caminhada em ritmo autosselecionado por mulheres adultas, previamente sedentárias.
\end{abstract}

Palavras-chave: exercício físico, envelhecimento, psicofisiologia.

\begin{abstract}
To compare the physiological, perception and affective responses during treadmill walking at a self-selected pace by previously sedentary women from three age groups. Methods: Sixty-six healthy women were assigned into three groups according to their age: Gl (20.0-25.0 yr, $\mathrm{n}=22)$, GII (30.0-35.0 yr, $\mathrm{n}=22)$ and GIII (40.0-45.0 yr, $n=22$ ). Each participant performed (i) an initial medical screening, anthropometric assessment and familiarization; (ii) an incremental treadmill test to determine O2max; and (iii) a 20-min treadmill walking bout at a self-selected pace. During the 20-min of treadmill walking at a self-selected pace, the physiological (oxygen uptake, $\mathrm{O} 2$ and heart rate, $\mathrm{HR}$ ) responses were continuously recorded. The perception (Borg-RPE for the overall body, 6-20) and affective (Feeling Scale) responses were measured every 5 min throughout the test. One-way ANOVA was used for statistical analysis $(p<0.05)$. Results: one-way ANOVA demonstrated that there were not significant differences in $\mathrm{O} 2$ and $\mathrm{HR}$ during a 20-min treadmill walking bout at a self-selected pace among the three age groups. However, the $\% \mathrm{O}_{2 \mathrm{Max}} \% \mathrm{O} 2 \mathrm{LV}, \% \mathrm{FC}_{\text {Max }}$ and \%FCLV were significantly higher in GIII compared with $\mathrm{Gl}$ and $\mathrm{Gll}(\mathrm{p}<0.05)$. Finally, the perception and affective responses during a 20 -min treadmill walking bout at a self-selected pace were similar among the three age groups. Conclusion: These findings demonstrated that the physiological but not the perception and affective responses to treadmill walking at a self-selected pace by sedentary women, did differ according to their age group.
\end{abstract}

Keywords: physical exercise, aging, psychophysiology. 


\section{INTRODUÇÃO}

A inatividade física tem sido reconhecida como um fator de risco modificável fundamental para as elevadas taxas de morbidade e mortalidade por doenças crônicas não transmissíveis verificadas em inúmeros países nas últimas décadas ${ }^{(1)}$. Apesar disso, uma considerável parcela da população adulta desses países é inativa ${ }^{(2-5)}$. No Brasil, por exemplo, estima-se que apenas $13 \%$ da população adulta realiza o mínimo recomendado de 30 minutos de exercício físico contínuo de intensidade moderada em três ou mais dias da semana. Ainda, somente 3,3\% dessa população realiza exercícios físicos com duração igual e/ou superior a 30 minutos em cinco ou mais dias da semana ${ }^{(3)}$. Essa elevada prevalência de inatividade física poderia ser resultante da associação de dois problemas distintos: a baixa taxa de engajamento inicial e a alta taxa de abandono em programas de exercício físico.

A prescrição de elevadas intensidades de exercício físico é um importante fator determinante para a diminuída aderência em programas de atividade física ${ }^{(6)}$. Estudos prévios demonstram haver uma associação direta entre intensidade de exercício físico e taxa de abandono ${ }^{(7-9)}$. Por exemplo, Dishman e Buckworth ${ }^{(7)}$ verificaram que intervenções baseadas na prescrição de uma intensidade leve (ou seja, um estímulo igual ou inferior a 50\% da capacidade máxima individual) apresentam taxa de aderência superior àquelas intervenções baseadas em prescrições de intensidades mais vigorosas. No entanto, independentemente dessas questões relativas ao debate dose-resposta entre intensidade e aderência, tem-se sugerido para que programas de exercício físico baseiem suas prescrições de intensidade dentro dos padrões mínimos requeridos para a ocorrência de modificações orgânicas benéficas à saúde. De acordo com o Colégio Americano de Medicina do Esporte $(\mathrm{ACSM})^{(10)}$, a prescrição de intensidades de exercício físico entre 50$85 \%$ do consumo máximo de oxigênio $\left(\mathrm{O}_{2 \text { Máx }}\right)$ e $55-90 \%$ da frequência cardíaca máxima $\left(\mathrm{FC}_{\text {Máx }}\right.$ ) são necessárias para se atingir significativas modificações orgânicas.

Apesar dessa necessidade da prescrição de uma adequada intensidade de exercício físico, estudos prévios demonstraram que sujeitos submetidos a intervenções de exercício físico tendem a autosselecionar intensidades divergentes daquelas intensidades previamente prescritas ${ }^{(6,8,11)}$. De um ponto de vista psicobiológico, essa autosseleção da intensidade baseia-se na produção preferencial de respostas perceptuais e afetivas (prazer/desprazer) positivas ${ }^{(12-14)}$. Ainda, estudos anteriores têm sugerido que seus participantes são capazes de autosselecionar estímulos fisiologicamente adequados para a ocorrência de modificações orgânicas benéficas à saúde ${ }^{(6,12,14,15)}$. Entretanto, recentes pesquisas demonstraram que essa intensidade de exercício físico preferida poderia ser fisiologicamente inadequada ${ }^{(13,16,17)}$, ou seja, um estímulo inferior aos parâmetros fisiológicos propostos pelo $\operatorname{ACSM}^{(10)}$. A variabilidade nos resultados desses estudos poderia ser devido a diversos fatores, incluindo diferenças relativas à massa e adiposidade

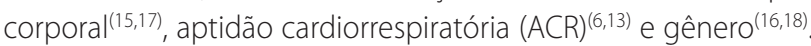

A idade têm sido indicada como outro possível fator contribuinte para essa variabilidade verificada nos resultados de estudos prévios envolvendo intensidades autosselecionadas ${ }^{(12,19)}$. Por exemplo, Malatesta et al. ${ }^{(19)}$ demonstraram que sujeitos apresentando idade mais elevada autosselecionavam uma menor velocidade de caminhada comparativamente aos indivíduos mais jovens (1,16 $\pm 0,09 \mathrm{~m} \cdot \mathrm{seg}^{-1} \mathrm{vs}$. $1,38 \pm 0,09 \mathrm{~m}_{\mathrm{seg}}{ }^{-1}$, respectivamente), porém apresentavam uma maior resposta fisiológica $\left(60,8 \pm 8,0 \%\right.$ vs. $42,9 \pm 5,0 \% \mathrm{O}_{2 \text { Máx }}$ respectivamente). Esses resultados têm sido corroborados por estudos anteriores ${ }^{(16,20)}$, os quais examinaram somente diferenças etárias relativas às respostas fisiológicas durante exercício físico em ritmo preferido, negligenciando assim a investigação das respostas perceptuais e afetivas. A produção preferencial de respostas perceptuais e afetivas positivas associadas ao exercício físico é fundamental para a ocorrência de um aumento na motivação intrínseca individual, e consequentemente, para a maior aderência aos programas de exercício físico ${ }^{(6,14)}$. No entanto, até o presente momento, nenhuma pesquisa buscou investigar conjuntamente as possíveis diferenças etárias relativas às respostas fisiológicas, perceptuais e afetivas durante exercício físico em ritmo autosselecionado. Neste contexto, o presente estudo buscou comparar as respostas fisiológicas, perceptuais e afetivas durante a realização de caminhada em ritmo autosselecionado por mulheres adultas de três diferentes faixas etárias.

\section{MÉTODOS}

\section{Participantes}

Participaram do presente estudo 66 mulheres com idades entre 20 e 45 anos. O recrutamento inicial dos possíveis participantes foi realizado através de anúncios pessoais e/ou impressos. Foram estabelecidos os seguintes critérios de inclusão: (a) condição de sedentária, indicado por uma participação < 30min de atividade física moderada em três ou mais dias da semana ${ }^{(10)}$; (b) nenhuma modificação ocorrida nos padrões de atividade física habitual durante os últimos seis meses; (c) totalidade das respostas negativas ao Physical Activity Readiness Questionnaire (PARQ); (d) nenhum histórico de distúrbios cardiovasculares, respiratórios, musculoesqueléticos e metabólicos; e (e) nenhum histórico de tabagismo.

Todos os sujeitos foram informados sobre os procedimentos utilizados, possíveis benefícios e riscos atrelados à execução do estudo, condicionando a sua participação de modo voluntário através da assinatura do Termo de Consentimento Livre e Informado. O protocolo de pesquisa foi delineado conforme as diretrizes propostas na Resolução 196/96 do Conselho Nacional de Saúde sobre pesquisas envolvendo seres humanos e posteriormente aprovado pelo Comitê de Ética em Pesquisa do Setor de Ciências da Saúde da Universidade Federal do Paraná (n 477.127.07.11).

\section{Delineamento experimental}

O presente estudo empregou um delineamento de pesquisa ex post facto $^{(21)}$. Cada participante completou três sessões experimentais, marcadas em diferentes dias. Durante a primeira sessão, todos os sujeitos realizaram uma avaliação médica, avaliação antropométrica e familiarização com os procedimentos experimentais. Por sua vez, a segunda sessão foi composta por um teste incremental máximo em esteira. Finalmente, a terceira sessão incluiu a realização de um teste de 20 minutos de caminhada em esteira em ritmo autosselecionado. Todos os participantes foram instruídos a não realizar exercício físico e a não ingerir quaisquer produtos contendo cafeína em um período de 24 horas precedentes ao início dos testes.

Com o intuito de melhor investigar a influência da idade sobre as respostas fisiológicas, perceptuais e afetivas durante caminhada em ritmo autosselecionado, os participantes foram categorizados como: (a) Gl: 20,0 - 25,0 anos, $n=22$; (b) Gll: 30,0 - 35,0 anos, $n=22$; ou (c) GIII: 40,0 - 45,0 anos, $n=22$. O número de sujeitos por grupo etário foi calculado com base em um nível de significância de 0,05, poder estatístico de 0,7 e magnitude de efeito grande $\left(f^{2}=0,35\right)$. Os dados descritivos dos sujeitos são apresentados nas tabelas 1 e 2 .

\section{Avaliação antropométrica}

Ao início da primeira sessão, todos os participantes foram submetidos a uma avaliação antropométrica realizada por um único pesquisador previamente treinado. As variáveis antropométricas massa corporal 
Tabela 1. Características antropométricas e demográfica dos participantes.

\begin{tabular}{c|c|c|c|c|c|c|c|c|c}
\hline \multirow{2}{*}{} & \multicolumn{2}{|c|}{ GI $(\boldsymbol{n}=\mathbf{2 2})$} & \multicolumn{3}{c|}{ GII $(\boldsymbol{n}=22)$} & \multicolumn{3}{c}{ GIII ( $=22)$} \\
\cline { 2 - 10 } & M & \pm & DP & M & \pm & DP & M & \pm & DP \\
\hline Idade (anos) & 22,0 & \pm & 1,5 & 33,0 & \pm & $1,8 *$ & 42,2 & \pm & 1,7 \#† \\
\hline $\begin{array}{c}\text { Massa corporal } \\
\text { (kg) }\end{array}$ & 61,7 & \pm & 9,5 & 62,1 & \pm & 8,8 & 68,2 & \pm & 12,4 \\
\hline Estatura (cm) & 161,5 & \pm & 5,9 & 162,8 & \pm & 6,5 & 161,5 & \pm & 9,0 \\
\hline IMC (kg.m-2) & 23,6 & \pm & 3,3 & 23,4 & \pm & 3,0 & 26,0 & \pm & $3,7 \dagger$ \\
\hline $\begin{array}{c}\text { \% Gordura } \\
\text { corporal }\end{array}$ & 26,3 & \pm & 2,5 & 27,6 & \pm & 4,4 & 31,7 & \pm & $4,5 \#+$ \\
\hline
\end{tabular}

M: média \pm DP: desvio padrão; IMC: índice de massa corporal; * Gl significativamente diferente de G\|l $(p<0,05)$ \# GI significativamente diferente de GIII $(p<0,05)$. † GII significativamente diferente de GIII $(p<0,05)$.

Tabela 2. Características fisiológicas, perceptuais e afetivas dos participantes.

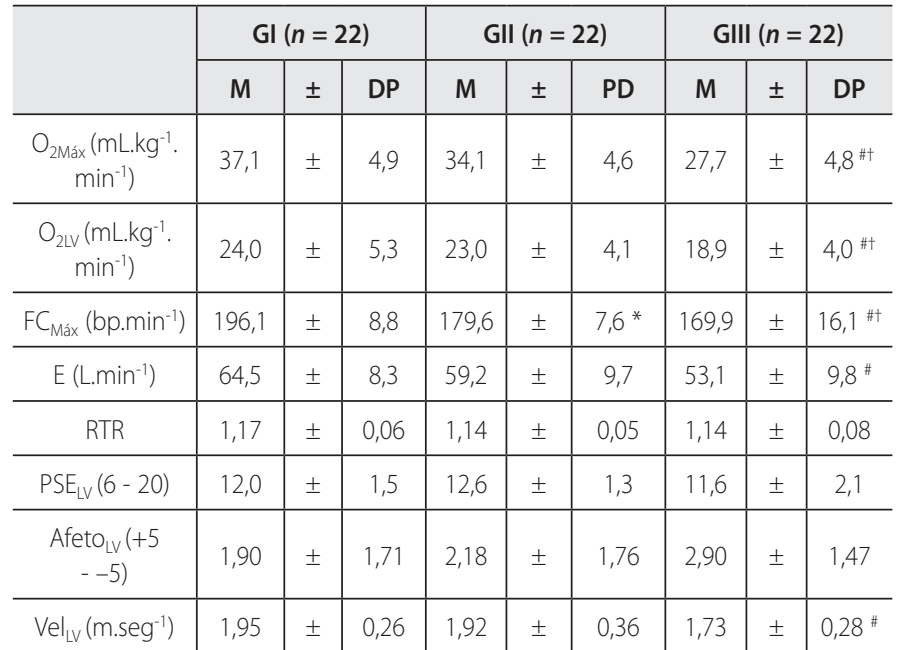

M: média $\pm \mathrm{DP}$ : desvio padrão; $\mathrm{VO}_{2 \mathrm{Max}}$ consumo máximo de oxigênio; $\mathrm{VO}_{2}$ : consumo de oxigênio no limiar ventilatório; $\mathrm{FC}_{\text {Max: }}$ : requência cardíaca máxima; VE: ventilação pulmonar; $\mathrm{RTR}$ : razão de troca respiratória; PSE: percepçăo subjetiva de esforço; LV: limiar ventilatório. " GI significativamente diferente de GII $(p<0,05) .{ }^{*} \mathrm{GI}$ significativamente diferente de $\mathrm{GIII}(p<0,05){ }^{\dagger} \mathrm{G}$ II significativamente diferente de $\mathrm{GIII}(p<0,05)$

(MC, em kg; balança marca Toledo ${ }^{\circledR}$, modelo 2096, São Paulo, Brasil) e estatura (EST, em cm; estadiômetro marca Sanny ${ }^{\circledR}$, modelo Standard, São Bernardo do Campo, Brasil) foram obtidas de acordo com os procedimentos descritos por Gordon et al. ${ }^{(22)}$. O índice de massa corporal (IMC, em kg/m²) foi calculado como a MC dividida pela EST ao quadrado. A densidade corporal foi estimada através do método de espessura de dobras cutâneas, de acordo com a equação proposta por Durnin e Womersley ${ }^{(23)}$. Posteriormente, o \% gordura corporal foi obtido mediante utilização da equação de Siri(24).

\section{Sessão de familiarização}

Durante a presente sessão, os participantes foram instruídos e familiarizados com o teste de exercício físico em esteira e o processo de seleção de um ritmo de caminhada preferido. Essa sessão de familiarização consistiu de uma caminhada inicial em esteira (model X Fit 7, Reebok Fitness $^{\top \mathrm{M}}$, Londres, Reino Unido), sem inclinação, durante $5 \mathrm{~min}$ no ritmo selecionado pelo sujeito. Após $10 \mathrm{~min}$ de repouso, esse procedimento de caminhada era então repetido. Ritmo autosselecionado foi definido como uma velocidade que o sujeito considerou confortável para a duração estipulada da atividade ${ }^{(13)}$. Durante essa sessão de familiarização e a sessão experimental, o marcador de velocidade da esteira, inicialmente fixo em $1,11 \mathrm{~m} \cdot \mathrm{seg}^{-1}\left(4,0 \mathrm{~km} \cdot \mathrm{h}^{-1}\right)$, foi ocultado para que os sujeitos não soubessem sua atual velocidade de caminhada ${ }^{(13,15)}$.

\section{Teste incremental máximo}

O teste incremental máximo foi realizado de acordo com o protocolo de Lind et al. ${ }^{(12)}$. Inicialmente, sujeitos realizaram um aqueci- mento padrão (caminhada em esteira por 5 min em uma velocidade de 1,11 m.seg ${ }^{-1}$ ). Após isso, o teste foi iniciado em uma velocidade de $1,11 \mathrm{~m} \cdot \mathrm{seg}^{-1}\left(4,0 \mathrm{~km} \cdot \mathrm{h}^{-1}\right)$, sem inclinação, durante $2 \mathrm{~min}$. Subsequentemente, a velocidade foi aumentada por 0,18 $\mathrm{m}_{\mathrm{seg}} \mathrm{s}^{-1}\left(0,64 \mathrm{~km} \cdot \mathrm{h}^{-1}\right)$ a cada 2 min até exaustão volitiva ${ }^{(12)}$. O critério para atingir o $\mathrm{O}_{2 \mathrm{Max}}$ deveria ser adequado a dois dos seguintes critérios: (a) um platô no $\mathrm{O}_{2}$ (variações $<150 \mathrm{~mL}$. $\mathrm{min}^{-1}$ nas últimas três médias consecutivas de 20 seg); (b) uma razão de troca respiratória (RTR) $\geq 1,10$; e (c) uma $F C_{\text {Max }}$ dentro de 10 bp. $\mathrm{min}^{-1}$ da FC predita pela idade. O limiar ventilatório (LV) foi determinado pelo método de equivalente ventilatório(25). Uma avaliação a posteriori para determinar o LV foi conduzida por dois avaliadores experientes.

A FC (bp. $\mathrm{min}^{-1}$ ) foi mensurada a cada 5 seg durante todo o teste usando um sistema de monitoramento Polar (Polar Electro ${ }^{\mathrm{TM}}$, Oy, Finlândia). Um sistema metabólico-respiratório de circuito aberto (True Max 2400, Parvo Medics ${ }^{\text {TM }}$, Salt Lake City, EUA) foi usado para mensurar $\mathrm{O}_{2}$, produção de dióxido de carbono $\left(\mathrm{CO}_{2}\right)$ e ventilação pulmonar (E, STPD) a cada 20seg durante todo o teste. Anteriormente a cada determinação do $\mathrm{O}_{2 \text { max }}$ o analisador de gases foi calibrado com concentrações conhecidas de gases.

\section{Teste de 20 minutos de caminhada em ritmo autosselecionado}

Após $72 \mathrm{~h}$ da condução do teste incremental máximo, participantes realizaram a sessão experimental. Instruções relativas à autosseleção do ritmo de caminhada foram apresentadas aos sujeitos. A sessão experimental foi composta de um teste de caminhada contínua em esteira durante $20 \mathrm{~min}$. Inicialmente, os sujeitos realizaram um aquecimento padrão (caminhada em esteira por $2 \mathrm{~min}$ em uma velocidade de 1,11 m. $\mathrm{seg}^{-1}$ ). Após isso, o teste foi iniciado em uma velocidade de $1,11 \mathrm{~m} \cdot \mathrm{seg}^{-1}$ $\left(4,0 \mathrm{~km} \cdot \mathrm{h}^{-1}\right)$, sem inclinação, por $2 \mathrm{~min}$. Durante os próximos $3 \mathrm{~min}$ do teste de caminhada de $20 \mathrm{~min}$, modificações na velocidade da esteira foram permitidas ad libitum. Subsequentemente, foram permitidas modificações na velocidade da esteira somente a cada $5 \mathrm{~min}$ do teste de 20min de caminhada ( $\min$ 5:00, 10:00 e 15:00).

As respostas metabólicas respiratórias e FC foram mensuradas a cada $1 \mathrm{~min}$ durante o teste usando os mesmos instrumentos e procedimentos do teste incremental máximo. Entretanto, somente os valores médios do último minuto de cada estágio foram considerados para a subsequente análise dos dados fisiológicos (ou seja, $\mathrm{O}_{2}$ e FC): (a) min 5:00, (b) $\min 10: 00$, (c) $\min 15: 00$ e (d) $\min 20: 00^{(12)}$.

\section{Mensurações de percepção subjetiva de esforço e afeto}

A escala de esforço percebido de Borg ${ }^{(26)}$ foi utilizada para mensurar a percepção subjetiva de esforço (PSE) durante exercício físico. Essa escala é composta de 15 pontos (6-20) de item único, com descritores verbais variando de "muito, muito leve" a "muito, muito pesado". Os descritores verbais baixo e alto foram ancorados durante teste incremental máximo. 0 escore de 7 (descritor verbal baixo) foi definido como a mais baixa intensidade de exercício físico, enquanto que o escore de 19 (descritor verbal alto) foi definido como a mais alta intensidade(27). Por sua vez, a escala de afeto de Hardy e Rejeski(27) foi empregada para mensurar a dimensão afetiva de prazer/desprazer. Comumente usada para a avaliação de respostas afetivas durante o exercício físico ${ }^{(12,14,15)}$, essa escala apresenta uma medida bipolar de 11 pontos, variando de -5 à +5 , com descritores verbais entre "pouco prazer" a "muito prazer", com um ponto zero "neutro". Durante os últimos 15 seg de cada estágio de 2 min do teste incremental máximo e a cada 5 min do teste de 20 min de caminhada em ritmo autosselecionado, ambas as escalas foram administradas aos sujeitos. Os participantes foram questionados para indicar a sua percepção subjetiva de esforço e sensação de prazer e desprazer nesses momentos particulares. 


\section{Procedimentos estatísticos}

Dados descritivos foram apresentados como média $(\mathrm{M}) \pm$ desvio padrão (DP). Uma ANOVA de um fator (faixa etária) foi utilizada para examinar as diferenças entre os grupos nas respostas fisiológicas, perceptuais e afetivas durante teste de $20 \mathrm{~min}$ de caminhada em ritmo autosselecionado. Além disso, uma ANOVA $3 \times 4$ (faixa etária $\times$ tempo) de medidas repetidas foi usada para determinar as mudanças através do tempo e entre grupos nas respostas fisiológicas, perceptuais e afetivas durante teste de 20min de caminhada em ritmo autosselecionado. Efeitos principais e de interação foram analisados através de uma ANOVA de um fator com correções de Bonferroni $(p<0,05)$. As correções epsilon de Greenhouse-Geisser foram usadas quando a premissa da esfericidade foi violada. O eta parcial quadrado $\left(\eta^{2}\right)$ foi usado para determinar a magnitude de efeito das respostas fisiológicas, perceptuais e afetivas. Todos os dados foram analisados usando SPSS 17.0 for Windows (SPSS, Inc., Chicago, EUA).

\section{RESULTADOS}

As características antropométricas dos participantes são apresentadas na tabela 1. Diferenças significativas entre os grupos etários foram verificadas somente para as variáveis idade $\left(F_{[2,63]}=778,906, p<0,001\right)$, $\operatorname{IMC}\left(F_{[2,63]}=4,071, p<0,001\right)$ e percentual de gordura corporal $\left(F_{[2,59]}\right.$ $=10,209, p<0,001)$.

As características fisiológicas, perceptuais e afetivas dos participantes são apresentadas na tabela 2. Decréscimos significativos no $\mathrm{O}_{2 \text { Máx }}$ $\left(F_{[2,63]}=22,255, p<0,001\right), \mathrm{FC}_{\text {Máx }}\left(F_{[2,63]}=29,153, p<0,001\right)$ e $\mathrm{E}_{\text {Máx }}\left(F_{[2,63]}\right.$ $=8,204, p<0,001)$ foram verificados entre os grupos etários. De modo similar, decréscimos significativos foram verificados no $\mathrm{O}_{2 \mathrm{LV}}\left(F_{[2,63]}=\right.$ $7,820, p<0,001)$ e na Vel $\mathrm{LV}_{\mathrm{LV}}\left(F_{[2,63]}=3,522, p<0,05\right)$ entre os grupos etários. Contudo, o \% $\mathrm{O}_{2 \mathrm{LV}}$ foi similar entre os grupos etários $(64,4 \pm$ $9,0,67,4 \pm 6,6$ e 68,4 $\pm 8,4$ para $G$ l, Gll, e Gll, respectivamente; $F_{[2,63]}=$ $1,439, p=0,245)$. Nenhuma diferença significativa foi verificada na PSE e afeto no LV entre os grupos etários.

As respostas fisiológicas, perceptuais e afetivas durante teste de 20min de caminhada em ritmo autosselecionado são apresentadas na tabela 3. As respostas fisiológicas relativas aos valores máximos, especificamente $\% \mathrm{O}_{2 \text { Máx }}\left(F_{[2,63]}=14,768, p<0,001\right)$ e \%FC Máx $_{(2,63]}=4,248, p<$ $0,05)$, tão bem como as respostas fisiológicas relativas aos valores no LV, especificamente $\% \mathrm{O}_{2 \mathrm{LV}}\left(F_{[2,63]}=6,017, p<0,05\right)$ e $\% \mathrm{FC}_{\mathrm{LV}}\left(F_{[2,63]}=2,544, p<\right.$ $0,05)$, demonstraram um aumento significativo entre os grupos etários. Em relação às respostas perceptuais e afetivas, nenhuma diferença foi verificada tanto na PSE como no afeto entre os grupos etários.
A figura 1 apresenta as respostas fisiológicas através do tempo durante teste de 20min de caminhada em ritmo autosselecionado. Em relação ao $\% \mathrm{O}_{2 \text { Máx }}$ a ANOVA $3 \times 4$ (faixa etária $\times$ tempo) de medidas repetidas demonstrou efeitos principais significativos na faixa etária $\left(F_{[2,42]}=16,541, p<0,001 ; \eta_{p}^{2}=0,441\right)$ e tempo $\left(F_{[3,63]}=27,580, p<0,001\right.$; $\left.\eta_{p}^{2}=0,568\right)$. De modo similar, os efeitos principais da faixa etária $\left(F_{[2,42]}=\right.$ $\left.5,693, p<0,05 ; \eta_{p}^{2}=0,213\right)$ e tempo $\left(F_{[2,197,46,139]}=91,420, p<0,001 ; \eta_{p}^{2}\right.$ $=0,813$ ) também foram significativos para o \% FC Máx. Contudo, nenhum efeito de interação faixa etária $\times$ tempo para ambas as variáveis foi observado. Em todos os períodos de tempo investigados (ou seja, min 5:00, 10:00, 15:00 e 20:00), diferenças significativas no $\% \mathrm{O}_{2 \text { Máx }}$ e \%FC $\mathrm{Fáx}$ entre Gl e GIII e também entre Gll e GIII foram verificadas $(p<0,05)$.

De modo similar às respostas fisiológicas, a ANOVA $3 \times 4$ (faixa etária $\times$ tempo) de medidas repetidas também demonstrou que os efeitos principais do tempo para PSE $\left(F_{[2,318148,680]}=70,602, p<0,001 ; \eta^{2}{ }_{p}=0,771\right)$ e afeto $\left(F_{[1,765,37,068]}=29,166 ; p<0,001 ; \eta_{p}^{2}=0,581\right)$ foram significativos (figura 1). Contudo, nenhum efeito principal da faixa etária ou de interação faixa etária $\times$ tempo para ambas as variáveis foi observado.

\section{DISCUSSÃO}

O objetivo deste estudo foi comparar as respostas fisiológicas, perceptuais e afetivas durante caminhada em ritmo autosselecionado por mulheres adultas de três diferentes faixas etárias. De acordo com a tabela 3, verificou-se que os sujeitos autosselecionaram uma intensidade de exercício físico adequada aos padrões estabelecidos pelo $\mathrm{ACSM}^{(10)}$ para a ocorrência de modificações orgânicas benéficas à saúde, independentemente da idade. Tal achado corrobora os resultados de prévios estudos ${ }^{(6,12,14,15)}$. Em relação às duas menores faixas etárias (Gl e GII), verificou-se que as intensidades autosselecionadas durante caminhada foram similares àquelas reportadas nos estudos de Spelman et al. ${ }^{(18)}$ e Pintar et al. ${ }^{(13)}$ (52\% e $54 \% \mathrm{O}_{2 \text { Máx }}$ respectivamente), os quais também utilizaram a caminhada como o modo de exercício físico em seus protocolos experimentais. Contudo, essas intensidades supracitadas são inferiores àquela verificada em GIII (tabela 3). A maior média etária desse grupo poderia ser um dos principais fatores contribuintes para a sua maior intensidade de exercício físico autosselecionada. Realmente, estudos anteriores demonstram que sujeitos com idades mais elevadas tendem a autosselecionar intensidades mais vigorosas $(16,17,20)$. Desse modo, pode-se acreditar que a idade apresenta uma influência significativa sobre a autosseleção da intensidade de exercício físico. Contudo, a influência de outros fatores, prioritariamente a $A C R^{(6)}$, não deve ser negligenciada.

Tabela 3. Respostas fisiológicas, perceptuais e afetivas durante teste de 20 min de caminhada em ritmo autosselecionado em esteira.

\begin{tabular}{|c|c|c|c|c|c|c|c|c|c|}
\hline & $\mathrm{GI}(n=22)$ & & & GII $(n=22)$ & & & GIII $(n=22)$ & & \\
\hline & M & \pm & DP & M & \pm & PD & M & \pm & DP \\
\hline $\begin{array}{c}\mathrm{O} 2 \text { (mL.kg-1. } \\
\text { min-1) }\end{array}$ & 19,9 & \pm & 3,5 & 19,6 & \pm & 3,3 & 18,8 & \pm & 4,2 \\
\hline \%O2Máx & 53,9 & \pm & 8,4 & 57,9 & \pm & 8,7 & 68,1 & \pm & 9,5 \#† \\
\hline$\% 02 \mathrm{LV}$ & 85,2 & \pm & 16,9 & 86,3 & \pm & 13,7 & 101,4 & \pm & 20,5 \#† \\
\hline FC (bp.min-1) & 143,4 & \pm & 17,0 & 134,9 & \pm & 11,5 & 136,3 & \pm & 19,3 \\
\hline \%FCMáx & 73,2 & \pm & 9,1 & 75,2 & \pm & 7,1 & 80,1 & \pm & $7,9 \#$ \\
\hline$\% F C L V$ & 93,5 & \pm & 12,4 & 92,6 & \pm & 8,2 & 98,8 & \pm & $7,9 \#$ \\
\hline PSE (6 - 20) & 11,6 & \pm & 1,4 & 12,0 & \pm & 1,4 & 12,4 & \pm & 1,0 \\
\hline Afeto $(+5--5)$ & 2,18 & \pm & 1,27 & 2,40 & \pm & 1,99 & 2,42 & \pm & 1,55 \\
\hline Vel (m.seg-1) & 1,69 & \pm & 0,19 & 1,61 & \pm & 0,20 & 1,63 & \pm & 0,19 \\
\hline
\end{tabular}

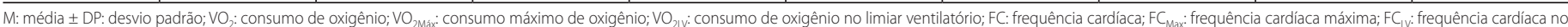

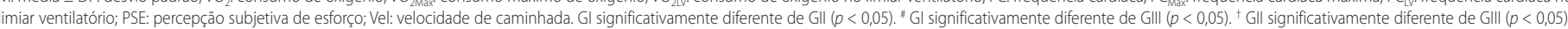




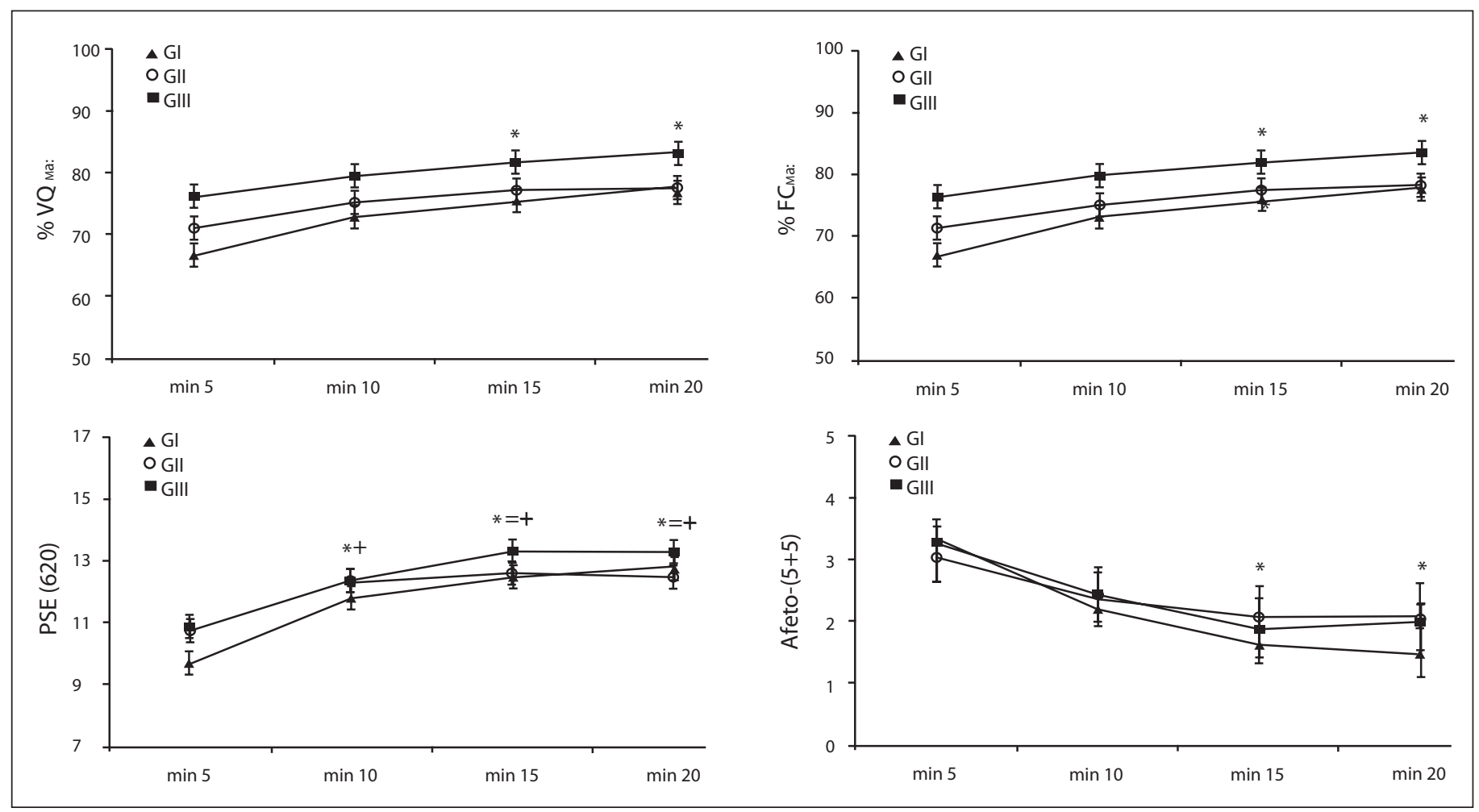

Figura 1. Respostas fisiológicas $\left(\% \mathrm{O}_{2 \text { Máx }}\right.$ e \% FC $\mathrm{Cáx}_{\text {Mx }}$, perceptuais (PSE) e afetivas através do tempo (min 5:00, 10:00, 15:00 e 20:00) durante teste de 20min de caminhada em ritmo autosselecionado em esteira. ${ }^{*}$ Diferença significativa do min 5:00 para Gl; ${ }^{*}$ Diferença significativa do min 5:00 para Gll; ${ }^{\dagger}$ Diferença significativa do min 5:00 para GIII.

A tabela 3 demonstra ainda que os participantes autosselecionaram intensidades de exercício físico próximas àquelas verificadas no ponto de transição de predominância entre os metabolismos aeróbico e anaeróbico $\left(\% \mathrm{O}_{2 \mathrm{LV}}\right.$ e \% FC $\left.\mathrm{LV}\right)$. Esses resultados são corroborados por estudos anteriores $^{(12,14,15)}$. A explicação para a proximidade entre as intensidades de exercício físico autosselecionadas durante teste de caminhada e àquelas intensidades associadas ao LV poderia derivar-se de suas respostas perceptuais e afetivas. De acordo com Rejeski(28), a realização de exercício físico em intensidades próximas ao LV estaria relacionada com as influências cognitiva e fisiológica periférica. Nesse sentido, pode-se esperar que as respostas perceptuais sejam heterogêneas, porém levemoderada (11-15) na escala de PSE de Borg. Ainda, conforme o modelo dose-resposta proposto por Ekkekakis et al. ${ }^{(29)}$, as respostas afetivas também podem apresentar-se heterogêneas, variando de pouco prazer até desprazer, conforme aspectos cognitivos individuais. Realmente, uma considerável variabilidade nas respostas perceptuais e principalmente afetivas associadas ao LV e a intensidade de exercício físico autosselecionada foi verificada no presente estudo, independentemente da idade (tabelas 2 e 3). Por outro lado, a realização de exercício físico em intensidades acima do LV estaria relacionada exclusivamente com a dominância de aspectos de origem fisiológica, porém não cognitiva(28). Desse modo, pode-se esperar a produção homogênea de respostas perceptuais pesadas e afetivas desprazerosas ${ }^{(29)}$, as quais são negativas à motivação intrínseca individual, e consequentemente, à aderência em programas de exercício físico ${ }^{(6,14)}$.

No presente estudo, verificou-se a produção de respostas perceptuais e afetivas positivas durante toda a realização da caminhada em ritmo autosselecionado, independentemente da idade (figura 1). De acordo com Ekkekakis et al.(29), essas respostas positivas poderiam contribuir para um aumento da motivação intrínseca individual. Tais achados são similares àquele verificado no estudo de Lind et al. ${ }^{(12)}$ (PSE, 10,9-13,7 e afeto 2,2-2,4), o qual empregou ambas as escalas em seu protocolo experimental. A administração das duas escalas (escala de
PSE e escala de sensação afetiva) contribui para uma investigação mais acurada sobre a experiência subjetiva durante realização de exercício físico, devido ao fato de PSE e afeto não serem considerados construtos isomorfos ${ }^{(27-29)}$. Realmente, em estudo realizado por Hardy e Rejeski(27), uma correlação negativa moderada $(r=-0,56)$ entre PSE e afeto foi verificada, similar àquela observada no presente estudo $(r=-0,61)$. Ainda, enquanto a PSE apresenta uma resposta linear durante exercício físico incremental, o afeto apresenta uma relação curvilinear ${ }^{(29)}$. Desse modo, dois indivíduos poderiam realizar exercício físico em uma mesma PSE (por ex., 15), porém um deles apresentar prazer pela atividade realizada, enquanto o outro apresentar desprazer.

O presente estudo apresenta limitações que necessitam ser citadas. Por exemplo, embora a mensuração do afeto tenha sido realizada mediante emprego da escala mais adequada para tal finalidade, o modelo circumplexo de afeto ${ }^{(29)}$ preconiza a utilização conjunta da escala de ativação percebida ${ }^{\left({ }^{30}\right)}$, a qual não foi empregada nesse estudo. Além disso, variáveis psicológicas situacionais (por ex., autoeficácia) e disposicionais (por ex., extroversão, neuroticismo, inibição comportamental), as quais poderiam estar associados a autosseleção da intensidade de exercício físico, não foram investigadas. Ainda, embora esse estudo tenha sido realizado em sujeitos com uma considerável variação etária (20,0-45,0 anos), esses resultados não deveriam ser generalizados para populações com idades inferiores e superiores, ou mesmo para sujeitos com características diferentes (homens, sujeitos fisicamente ativos, etc) ou portadores de condições especiais de saúde (diabéticos, hipertensos, obesos, etc). Finalmente, esse estudo foi realizado em ambiente laboratorial com a utilização de uma esteira motorizada, não favorecendo assim a generalização desses resultados para situações não laboratoriais em ambientes externos.

De acordo com os resultados do presente estudo, pode-se concluir que as respostas fisiológicas, porém não as respostas perceptuais e afetivas durante caminhada em ritmo autosselecionado por mulheres sedentárias, diferem entre as faixas etárias. Contudo, essas 
respostas fisiológicas são adequadas para a ocorrência de modificações orgânicas benéficas à saúde, independentemente da faixa etária. Ainda, a produção de respostas perceptuais e afetivas positivas durante caminhada em ritmo autosselecionado verificada em todas as faixas etárias poderia contribuir uniformemente para uma aumentada motivação intrínseca individual e aderência à programas de exercício físico. Entretanto, uma considerável variabilidade foi observada nessas respostas perceptuais e afetivas, e a investigação da origem dessa variabilidade é necessária em pesquisas futuras. Finalmente, os achados do presente estudo trazem futuras contribuições teóricas e práticas. De um ponto de vista teórico, indica a necessidade para que delineamentos experimentais futuros envolvendo a autosseleção do ritmo de exercício físico façam o controle da variável idade. De um ponto de vista prático, demonstra que a autosseleção de exercício físico é capaz de proporcionar a ocorrência de um estímulo fisiológico adequado para a ocorrência de modificações orgânicas benéficas à saúde e também de produzir respostas perceptuais e afetivas positivas, as quais contribuiriam para uma menor probabilidade de abandono em programas de exercício físico.

\section{AGRADECIMENTOS}

O presente estudo foi parcialmente financiado pelo Istituto per il Credito Sportivo, Italia (C.F.Buzzachera) e pela Coordenação de Aperfeiçoamento de Pessoal de Nível Superior, Brasil (H. M. Elsangedy e K. Krinski).

Todos os autores declararam não haver qualquer potencial conflito de interesses referente a este artigo.

\section{REFERÊNCIAS}

1. World Health Organization. Obesity: preventing and managing the global epidemic: report of a WHO consultation. Geneva: Technical Report Series, 2000.

2. Dowda M, Ainsworth BE, Addy CL, Saunders R, Riner W. Correlates of physical activity among U.S. young adults: 18 to 30 years of age from NHANES III. Ann Behav Med 2003;26:15-23.

3. Monteiro CA, Conde WL, Matsudo SM, Matsudo VR, Bonsenor IM, Lotufo PA. A descriptive epidemiology of leisure-time physical activity in Brazil, 1996-1997. Rev Panam Salud Publica 2003;14:246-54.

4. Oppert JM, Thomas F, Charles MA, Benetos A, Basdevant A, Simon C. Leisure-time and occupational physical activity in relation to cardiovascular risk factors and eating habits in French adults. Public Health Nutr 2006;9:746-54.

5. Monda KL, Gordon-Larsen P, Stevens J, Popkin BM. China's transition: the effect of rapid urbanization on adult occupational physical activity. Soc Sci Med 2007;64:858-70.

6. Dishman RK, Farquhar RP, Cureton KJ. Responses to preferred intensities of exertion in men differing in activity levels. Med Sci Sports Exerc 1994;26:783-90.

7. Dishman RK, Buckworth J. Increasing physical inactivity: a quantitative synthesis. Med Sci Sports Exerc 1996;28:706-19.

8. Cox KL, Burke V, Gorely T, Beilin L, Puddey IB. Controlled comparison of retention and adherence in home- vs center-initiated exercise interventions in women ages $40-65$ years: The S.W.E.A.T. Study (Sedentary Women Exercise Adherence Trial). Prev Med 2003;36:17-29.

9. Duncan GE, Anton SD, Sydeman SJ, Newton Jr RL, Corsica JA, Durning PE, et al. Prescribing exercise at varied levels of intensity and frequency: a randomized trial. Arch Intern Med 2005;165:2362-9.

10. American College of Sports Medicine. ACMS's guidelines for exercise testing and prescription. Philadelphia: Lippincott Williams and Wilkins, 2000

11. King AC, Haskell WL, Taylor CB, Kraemer HC, DeBusk RF. Group vs home-based exercise training in healthy older men and women: a community-based trial. JAMA 1991;266:1535-42.

12. Lind E, Joens-Matre RR, Ekkekakis P. What intensity of physical activity do previously sedentary middleaged women select? Evidence of a coherent pattern from physiological, perceptual, and affective markers. Prev Med 2005;40:407-19.

13. Pintar JA, Robertson RJ, Kriska AM, Nagle E, Goss FL. The influence of fitness and body weight on preferred exercise intensity. Med Sci Sports Exerc 2006;38:981-8.

14. Parfitt G, Rose EA, Burgess WM. The psychological and physiological responses of sedentary individuals to prescribed and preferred intensity exercise. Br J Health Psychol 2006;11:39-53.

15. DaSilva SG, Guidetti L, Buzzachera CF, Elsangedy HM, Colombo H, Krinski K, et al. The influence of adiposity on physiological, perceptual, and affective responses during walking at a self-selected pace. Percept Motor Skills 2009 (In Press).

16. Porcari JP, Ward A, Morgan WP, Ebbeling C, O'Hainly S, Rippe JM. Exercise intensity at a self-selected or preferred walking pace. J Cardiac Rehab 1988;8:398

17. Hills AP, Byrne NM, Wearing $S$, Armstrong T. Validation of the intensity of walking for pleasure in obese adults. Prev Med 2006;42:47-50.

18. Spelman CC, Pate RR, Macera CA, Ward DS. Self-selected exercise intensity of habitual walkers. Med Sci Sports Exerc 1993;25:1174-9.

19. Malatesta D, Simar D, Dauvilliers Y, Candau R, Saad HE, Prefaut C, et al. Aerobic determinants of the decline in preferred walking speed in healthy, active 65- and 80-years-old. Eur J Physiol 2004;447:915-21.

20. Buzzachera CF, Elsangedy HM, Hallage T, DaSilva SG. Parâmetros fisiológicos e perceptivos durante caminhada de intensidade preferida por mulheres adultas, previamente sedentárias. Rev Bras Cineantr Desempenho Hum 2007;9:170-6.

21. Thomas JR; Nelson JK. Research methods in physical activity. Champaign: Human Kinetics Books, 2001.

22. Gordon CC, Chumlea WC, Roche AF. Stature, recumbent length and weight. In: Lohman TG, Roche AF, Martorell R (eds). Anthropometric standardization reference manual. Champaign: Human Kinetics Books, 1988.

23. Durnin JCG, Womersley J. Body fat assessed from total body density and its estimation from skinfold thickness: measurements on 481 men and women aged from 16 to 72 years. Brit J Nutr 1974;32:77-97.

24. Siri WE. Body composition from fluid space and density. In: Brozek J, Hanschel A. (eds.) Techniques for measuring body composition. Washington: National Academy of Science, 1961.

25. Caiozzo VJ, Davis JA, Ellis JF, Azus JL, Vandergriff R, Prietto CA, et al. A comparison of gas exchange indices used to detect the anaerobic threshold. J Appl Physiol 1982;53:1184-9.

26. Borg GAV. Psychophysical bases of perceived exertion. Med Sci Sports Exerc 1982;14:377-81.

27. Hardy CJ, Rejeski WJ. Not what, but how one feels: The measurement of affect during exercise. J Sport Exerc Psychol 1989;11:204-317.

28. Rejeski WJ. The perception of exertion: a social psychophysiological integration. J Sport Psychol 1981;4:305-20

29. Ekkekakis P, Hall EE, Petruzzello SJ. Variation and homogeneity in affective responses to physical activity of varying intensities: an alternative perspective on dose-response based on evolutionary considerations. J Sports Sci 2005;23:477-500.

30. Russell JA. A circumplex model of affect. J Pers Soc Psychol 1980;39:1161. 